\title{
Anomalous bubble propagation in elastic tubes
}

\author{
Alexandra Heap and Anne Juel ${ }^{a)}$ \\ Manchester Centre for Nonlinear Dynamics and School of Mathematics, Alan Turing Building, \\ The University of Manchester, Oxford Road, Manchester M13 9PL, United Kingdom
}

(Received 28 April 2008; accepted 3 July 2008; published online 13 August 2008)

\begin{abstract}
Airway reopening is an important physiological event, as exemplified by the first breath of an infant that inflates highly collapsed airways by driving a finger of air through its fluid-filled lungs. Whereas fundamental models of airway reopening predict the steady propagation of only one type of bubble with a characteristic rounded tip, our experiments reveal a surprising selection of novel bubbles with counterintuitive shapes that reopen strongly collapsed, liquid-filled elastic tubes. Our multiple bubbles are associated with a discontinuous relationship between bubble pressure and speed that sets exciting challenges for modelers. (C) 2008 American Institute of Physics. [DOI: 10.1063/1.2963495]
\end{abstract}

From lab-on-a-chip devices ${ }^{1}$ to oil extraction from porous rocks, ${ }^{2}$ bubbles that displace a more viscous fluid exhibit rounded tips because of the moderating influence of surface tension. The first breath of an infant that inflates and reopens its highly collapsed airways by driving a finger of air through its fluid-filled lungs ${ }^{3-5}$ is an example of such a twophase displacement flow that interacts with the elastic airway. During artificial ventilation to reopen peripheral airways in diseased lungs, the avoidance of tissue damage relies fundamentally on the understanding of the relationship between bubble pressure and speed. ${ }^{6,7}$ Whereas previous models of airway reopening predict the steady propagation of only one type of bubble with a continuous relationship between bubble pressure and speed, and a characteristic rounded tip, ${ }^{8,9}$ our bench-top experiments reveal a surprising selection of novel bubbles with counterintuitive shapes (Fig. 1), which propagate steadily to reopen a strongly collapsed, liquid-filled elastic tube. These include anomalous features such as sharp points and cusp-shaped protrusions that are not smoothed out by surface tension forces on the lengthscale of the tube radius (but presumably on a much smaller lengthscale). These protrusions are reminiscent of the cusp singularities in free-surface entrainment flows ${ }^{10}$ and the freesurface cusps $^{11}$ and tips ${ }^{12}$ that can be formed when withdrawing fluid through a small orifice. Moreover, our multiple bubbles are associated with a discontinuous relationship between bubble pressure and speed that sets exciting challenges for modelers.

Bubble propagation in a liquid-filled, collapsed elastic tube is governed by the competition between viscous, surface tension, and elastic forces, through the variation of the capillary number and the level of tube collapse. The capillary number, $\mathrm{Ca}=\mu U / \sigma^{*}$, where $U$ is the bubble speed and $\mu$ and $\sigma^{*}$ the dynamic viscosity and surface tension of the lining liquid, respectively, corresponds to the ratio of viscous to surface tension forces. The dimensionless cross-sectional area $A / A_{0}$, where $A$ is the cross-sectional area of the collapsed tube, $A_{0}=\pi R^{2}$, and $R$ is the inner radius of the undeformed tube, is associated with the pressure of elastic collapse via a constitutive relation known as the "tube law,"

$\overline{\left.{ }^{a}\right) E l e c t r o n i c ~ m a i l: ~ a n n e . j u e l @ m a n c h e s t e r . a c . u k . ~}$ which was measured in the laboratory. ${ }^{13}$ The limit of high levels of collapse is addressed in this paper with most of the experiments performed for $A / A_{0} \leq\left(A / A_{0}\right)_{\text {owc }}$, where $\left(A / A_{0}\right)_{\text {owc }}=0.18$ corresponds to the level of collapse for which the opposite walls of the cross section make point contact [see Fig. 2(a)]. Although the collapse of airways in diseased lungs has not been documented quantitatively, numerical simulations of nonaxisymmetric collapse through fluid-elastic instabilities have shown that occluding liquid bridges can be formed from very small volumes of liquids. These models suggest that high levels of tube collapse are likely to be reached locally in the lungs. ${ }^{14}$

Fluid-structure interaction is quantified by $\sigma=\sigma^{*} /(R K)$, the ratio of surface tension to elastic forces, where $K$ denotes the bending stiffness of the tube. Previous experiments were performed on tubes of negligible bending stiffness, where the fluid-structure interaction arose from the large tension imposed on the end of the tube, ${ }^{15}$ and the tube retained an approximately uniform cross section. Only one type of bubble with a rounded tip was observed even for strongly collapsed tubes. A "ribbed" bubble front was noted at the onset of experiments in a two-dimensional geometry, ${ }^{16}$ but the nonuniformities decayed as the bubble front propagated. These bubbles are accurately captured by two-dimensional models ${ }^{17}$ which find that they propagate by peeling apart the tube walls and that their dimensionless pressure on the capillary scale (relative to atmospheric pressure), $P$, increases approximately linearly with $\mathrm{Ca}$. Qualitatively similar peeling bubbles exhibiting a rounded tip were also obtained from three-dimensional simulations of moderately collapsed airways, which couple nonlinear shell theory to the Stokes flow equations in the absence of gravity. ${ }^{9}$ However, the range of levels of collapse studied was limited to $0.39 \leq A / A_{0} \leq 0.62$.

We performed airway reopening experiments with a 1.0 $\mathrm{m}$ long piece of translucent extruded silicone tubing (Primasil Silicones Ltd., inner radius $R=4.99 \pm 0.16 \mathrm{~mm}$ and wall thickness $h=0.57 \pm 0.08 \mathrm{~mm}$ ) filled with paraffin oil (dynamic viscosity $\mu=2.04 \times 10^{-1} \mathrm{~kg} \mathrm{~m}^{-1} \mathrm{~s}^{-1}$, density $\rho=8.64$ $\times 10^{3} \mathrm{~kg} \mathrm{~m}^{-3}$, and surface tension $\sigma^{*}=2.9 \times 10^{-2} \mathrm{~N} \mathrm{~m}^{-1}$ measured in the laboratory at $18{ }^{\circ} \mathrm{C}$ ). The reproducibility of the results was ensured by handling the fragile elastic tube with extreme care and replacing the tube every three days, so 



FIG. 1. Examples of top-view snapshots of the main reopening bubbles (outlined with a solid line). The arrow indicates their direction of propagation. The horizontal line in the top picture represents the vertical plane of symmetry of the peeling bubble. In addition to this common bubble, we observe the selection of steady anomalous bubbles in the limit of strong tube collapse. These include asymmetric bubbles, double-tipped, and, most surprisingly, pointed bubbles. $A / A_{0}=0.26$ and $\mathrm{Ca}=0.13$ for the symmetric bubble, and $A / A_{0}=0.13$ for the others: $\mathrm{Ca}=0.59$ for the asymmetric bubble, $\mathrm{Ca}=1.03$ for the double-tipped bubble, and $\mathrm{Ca}=3.28$ for the pointed bubble.

that the slow permeation of the oil into the thin silicone wall did not alter the elastic properties of the material. The tube was uniformly collapsed by compressing it between two horizontal plates separated by a constant gap that could be adjusted to within $2 \%$ of the height of the collapsed tube to yield levels of collapse in the range of $0.02 \leq A / A_{0} \leq 0.41$. On completion of this procedure, the tube relaxed rapidly into its equilibrium dumbbell shape [see Fig. 2(a)]. A constant flow of nitrogen was injected into the collapsed tube by triggering the solenoid valve located at its inlet, resulting in the steady propagation of a long bubble. This trigger also initiated the pressure measurements and the video capture. The experiment was discontinued by opening the downstream valve when the tip of the propagating air bubble reached the end of the tube, in order to avoid permanent deformation due to overinflation. The nitrogen flow was controlled by a fine needle valve to range between 50 and $1400 \mathrm{~cm}^{3} \mathrm{~min}^{-1}$ and could be replicated to within 3\%. Pressure measurements (relative to atmospheric pressure) were taken with a differential pressure sensor (Honeywell, $\pm 5 \mathrm{~cm}$ $\mathrm{H}_{2} \mathrm{O}$ ) upstream of the collapsed tube, and the small pressure drop that occurred along the rigid line between the sensor and the inlet of the elastic tube was systematically subtracted in order to yield measurements of the pressure inside the elastic tube. Dewetting of the paraffin oil film was not observed during bubble propagation even in the most collapsed tubes, and all the results were qualitatively reproduced with silicone oil that fully wets the tube walls. (a)

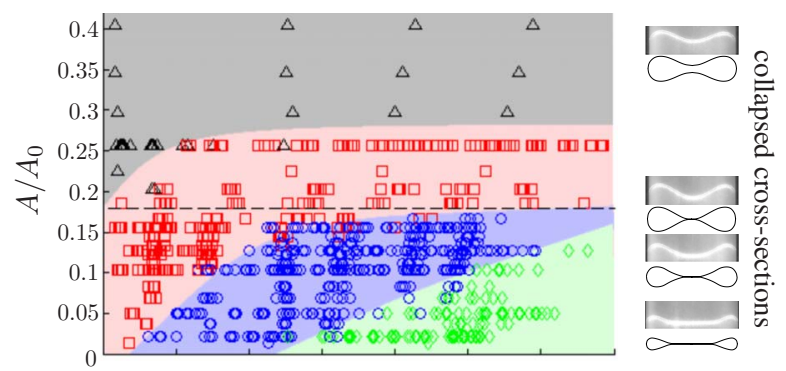

(b)

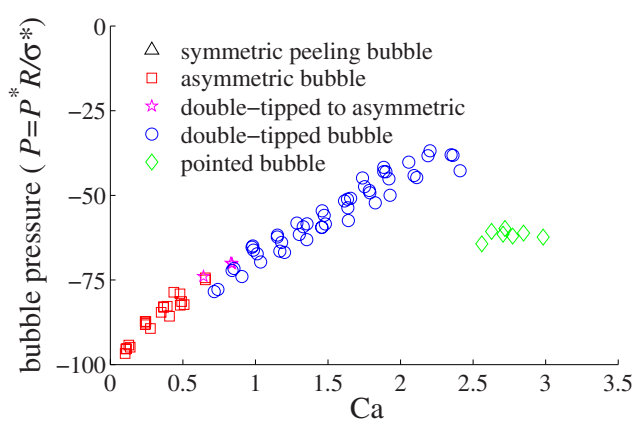

FIG. 2. (Color online) (a) Phase diagram in terms of the dimensionless bubble speed $\left(\mathrm{Ca}=\mu U / \sigma^{*}\right.$, where $U$ is the bubble speed and $\mu$ and $\sigma^{*}$ are the viscosity and surface tension of the liquid, respectively) and the dimensionless cross-sectional area of the collapsed tube $\left(A / A_{0}\right.$, where $A_{0}=\pi R^{2}$ and $R$ is the inner radius of the tube). Examples of collapsed cross sections (experimental top view and sketch) are given on the right hand side for $A / A_{0}=0.36,0.18,0.13$, and 0.04 from top to bottom, respectively. $A / A_{0}$ $=0.18$ is marked with a dashed horizontal line as it is the threshold below which the opposite walls of the cross section are in contact. The different bubble types are observed in well-defined regions of parameter space that are colored to guide the eye. (b) Relationship between the bubble pressure (relative to atmospheric pressure) and the dimensionless bubble speed $(\mathrm{Ca})$ for $A / A_{0}=0.10$. The pressure increases approximately linearly with $\mathrm{Ca}$ and the pressure transition between asymmetric and double-tipped bubble is continuous. The transition to pointed bubbles is accompanied by a significant pressure step that decreases with increasing tube collapse. This challenges the established notion of a continuous relationship between pressure and bubble speed in airway reopening.

In our experiments, the ratio of surface tension to elastic forces was $\sigma=(3.4 \pm 0.3) \times 10^{-2}$, where the bending stiffness of the tube was determined by rescaling the pressure required to collapse the tube to opposite wall contact measured in the laboratory with its value predicted by thin shell theory. ${ }^{18}$ The value of $\sigma$ was small because the tube was selected to be sufficiently stiff so that it would not deform under its own weight when empty. In the highly compliant peripheral airways of the lung, the value of $\sigma$ is approximately two orders of magnitude larger. However, the variation of $\sigma$ within this range only shifts the $P$-Ca curves without altering them qualitatively, as shown by three-dimensional numerical simulations. ${ }^{9,13}$ This gives an indication of the robustness of the peeling solution and suggests that our findings may also be relevant to a wider range of $\sigma$ up to pulmonary airway values. There is an inherent asymmetry about the horizontal centerplane in our system due to gravity, whose main influence on the peeling bubble at moderate levels of collapse $\left(A / A_{0}=0.41\right)$ is to shift the value of the bubble pressure. ${ }^{13}$ The influence of gravity forces on the reopening dynamics decreases with increasing level of collapse, as the thickness of the liquid lining in the tube is reduced. The Bond number, 
(a)

(b)
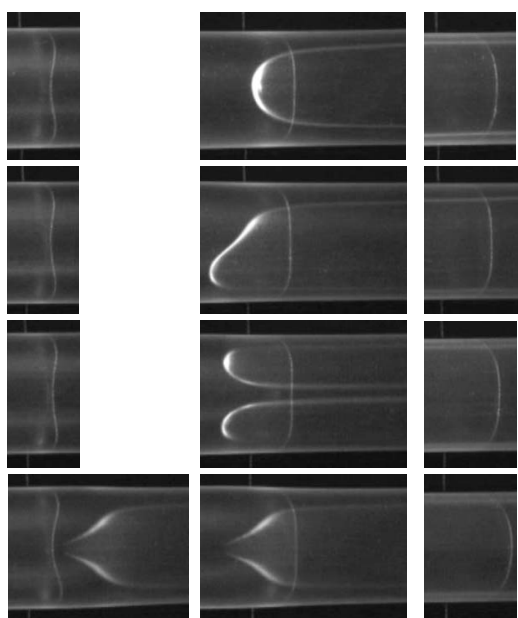

$A / A_{0}=0.26$

$\mathrm{Ca}=0.13$

(c)
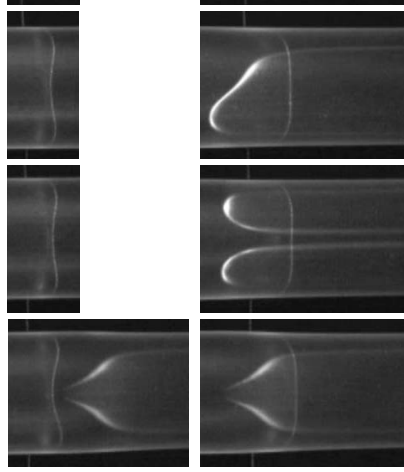

$A / A_{0}=0.13$

$\mathrm{Ca}=0.59$

$A / A_{0}=0.13$

$\mathrm{Ca}=1.03$

(d)

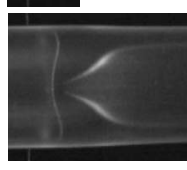



$A / A_{0}=0.13$

$\mathrm{Ca}=3.28-3.42$

FIG. 3. Laser sheet outlines of the top half of the tube before, during, and after the passage of the bubble: (a) Symmetric bubble, (b) asymmetric bubble, (c) double-tipped bubble, and (d) pointed bubble $(\mathrm{Ca}=3.42$ and 3.28 in the first and second pictures, respectively). The pictures are aligned vertically on the undeformed laser line. Note that the tube reopens to an elliptical shape as the bubble tip passes the laser line in all four cases. This implies that the fingers of the double-tipped bubble are separated by liquid, which was displaced by the propagating fingers to fill the central part of the cross section. Also the central part of the cross section is not reopened effectively by the infiltration of the cusped protrusion into the region of opposite wall contact.

Bo $=\rho g h^{2} /\left(4 \sigma^{*}\right)$, which measures the ratio of gravity to surface tension forces based on the film thickness $h$, takes uniformly small values in the range of $0.03 \leq \mathrm{Bo} \leq 0.07$ for $0.02 \leq A / A_{0} \leq\left(A / A_{0}\right)_{\text {owc }}$ compared to $\mathrm{Bo}=0.5$ for $A / A_{0}$ $=0.41$. Furthermore, the effect of inertial forces is negligible over the range of parameters investigated here. . $^{13,19}$

The deformation of the top half of the tube was outlined with a laser sheet shone at an angle of $50^{\circ}$ from the vertical [see Fig. 2(a)]. An aerial view camera, located $65 \mathrm{~cm}$ downstream of the inlet, captured an $8.5 \mathrm{~cm}$ long top view of the reopening tube illuminated from below, with a resolution of $0.12 \mathrm{~mm} / \mathrm{pixel}$. Movies of the advancing reopening front were analyzed to yield a measurement of the speed of the propagating air bubble, averaged over the length of the visualization window. Edge detection was performed on the topview bubble images, which exhibited remarkable contrast despite the unavoidable light scattering caused by the translucent nature of the tube.

840 experiments are summarized in Fig. 2(a) in a phase diagram in terms of $\mathrm{Ca}$ and $A / A_{0}$. We find three new types of bubbles that are each selected within clearly defined parameter ranges: "Asymmetric," "double-tipped," and "pointed" (see Fig. 1). Each bubble included in Fig. 2(a) remained stable as it propagated steadily along the length of the experimental tube. Laser line visualizations of the top half of the tube before, during, and after the passage of the bubble in Fig. 3 indicate that in all cases, the cross section was reopened partially to an approximately elliptical shape by the bubble tip. However, the tube required longer distances to reopen to its final height, which depended on the type of bubble and the values of $A / A_{0}$ and $\mathrm{Ca}$.

The supercritical breaking of the bubble symmetry about the vertical center plane of the tube offers an exciting chal- lenge for modelers; asymmetric bubbles are selected instead of the familiar symmetric peeling bubble for collapsed crosssectional areas as large as $A / A_{0}=0.26$. Moreover, the asymmetry of the tip was enhanced with both increasing $\mathrm{Ca}$ and levels of collapse (i.e., decreasing $\left.A / A_{0}\right) .{ }^{20}$ Note that the asymmetric bubble is selected at small values of $\mathrm{Ca}$, which yield limited tube reopening. In addition, the dumb-bellshaped cross section needs to be sufficiently nonuniform, forcing the bubble to propagate in either lobe where viscous shear is less than in the thinner central part of the cross section [see Fig. 2(a)]. The opposite walls of the collapsed cross section make contact for $A / A_{0} \leq\left(A / A_{0}\right)_{\text {owc }}=0.18$, and below this threshold only novel bubbles are observed (see Fig. 1). As the influence of surface tension decreases when $\mathrm{Ca}$ increases or $A / A_{0}$ is reduced [i.e., the pressure of elastic collapse steeply increases, see Fig. 2(a) for examples of collapsed cross sections], successive discontinuous transitions take place from asymmetric to double-tipped bubbles and then from double-tipped to pointed bubbles.

Double-tipped bubbles exhibit two separate precursor fingers that initiate the reopening of the side lobes of the collapsed tube independently (see Fig. 1). Similarly to the asymmetric bubble, they favor propagation in the lobes of the cross section where viscous shear is less than in the thinner central part. Similar bubbles have been obtained in static three-dimensional menisci simulations $(\mathrm{Ca}=0)$ under partial wetting conditions when the tube was collapsed to a dumbbell shape by external pressure. ${ }^{21}$ In these simulations, however, a reflection symmetry about the vertical center plane of the tube was imposed. In our experiments, we find bubbles that are asymmetric about this center plane at values of $\mathrm{Ca}$ smaller than those required for double-tipped bubbles to be selected for all values of $A / A_{0} \leq\left(A / A_{0}\right)_{\text {owc }}$ [see Fig. 2(a)]. For $A / A_{0} \geq\left(A / A_{0}\right)_{\text {owc }}$, the bubbles are symmetric at the lowest values of $\mathrm{Ca}$ investigated. The fingers of the doubletipped bubble merge at a distance behind the tip that is bound by the length of the transition region from collapsed to reopened tube and depends on the value of $\mathrm{Ca}$ and $A / A_{0}$. The tube adopts an approximately elliptical shape right behind the tips rather than the strongly collapsed shape seen ahead of the bubble [see Fig. 3(c)]. This indicates that the fingers of the double-tipped bubble are separated by liquid that is displaced by the finger tips to fill the central part of the cross section. The length of the fingers was usually of the order of one tube diameter, but for values of Ca close to the threshold below which asymmetric bubbles were selected, the length of the fingers rose sharply, and stable double-tipped bubbles were observed with precursor fingers measuring up to ten tube diameters in length.

Pointed bubbles were the most surprising interfaces observed (see Fig. 1). Indeed, the existence of the thin precursor finger defies intuition as it infiltrates the most collapsed central part of the cross section, where the upper and lower tube boundaries are in contact, without reopening it effectively [see Fig. 3(d)]. The formation of this cusp-shaped protrusion of approximately constant length relies upon the dominance of viscous and elastic forces. Difficulties associated with bubble and flow visualization in the translucent tube did not allow to resolve the tip of the protrusion accu- 


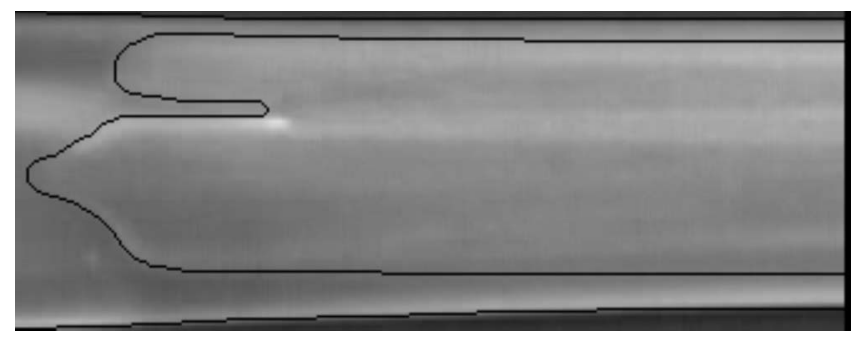

FIG. 4. Steadily propagating reopening bubble for nearly total initial collapse $\left(A / A_{0}=0.02\right.$ and $\left.\mathrm{Ca}=1.8\right)$.

rately nor the recirculations ahead of the bubble, and thus, analogies with the formation of other interfacial cusps ${ }^{10-12}$ could not be explored. Note that strong recirculation flows may be responsible for focusing the interfacial flow to induce the pointed bubble state. This type of recirculation flow has been found at low $\mathrm{Ca}$ in both elastic ${ }^{17}$ and rigid tubes, ${ }^{22,23}$ but usually disappears when $\mathrm{Ca}$ increases. For the strongest levels of collapse, $A / A_{0}<0.04$, more complex bubbles were observed, but they systematically included features of one or more novel bubbles shown in Fig. 1, suggesting that these are robust reopening bubbles. An example is shown in Fig. 4, where one of the fingers in a double-tipped bubble is itself a pointed bubble.

The bubble pressure (relative to atmospheric pressure) remains negative as shown in Fig. 2(b) for $A / A_{0}=0.10$, indicating that all the propagating bubbles only reopen the tube partially. This is because of the relatively small ratio of surface tension to elastic forces. ${ }^{13,19}$ The monotonic growth of the bubble pressure with $\mathrm{Ca}$ and the small volumes of fluid displaced ahead of the bubble tip suggest that all our novel bubbles are of "peeling" type. The asymmetric and doubletipped bubbles exhibit similar linear dependences on $\mathrm{Ca}$, and thus, the pressure transition between these two types of bubbles is smooth, and moreover, it cannot be identified from the pressure graph alone. A narrow region of bistability that was not detailed in Fig. 2(a) in the interests of clarity is shown in Fig. 2(b). Within this narrow range of $\mathrm{Ca}$, a doubletipped bubble would initially form before suddenly switching to an asymmetric bubble during the course of a reopening experiment where all the parameters were held fixed. This transition appears to be driven by the background noise in the experiment. Both states may be stable within a narrow region of the parameter plane, but alternatively, it could be that fluctuations shift the experimental parameters across their threshold values, thus leading to the switches between bubbles. Although the pressure curve is approximately linear, it differs quantitatively from the symmetric peeling state measured above the point of opposite wall contact, e.g., for $A / A_{0}>0.18$. Remarkably, a significant pressure step (of approximately $25 \%$ of the total pressure range) characterizes the transition from double-tipped to pointed bubbles, challenging the established notion that the relationship between pressure and speed is continuous. ${ }^{8,9}$

The discovery of multiple bubbles in the limit of strong tube collapse suggests that the reopening of a fluid-filled elastic tube exhibits a wide range of dynamics, which have so far remained unexplored, and could be of significance to pulmonary airway reopening. A detailed study of the transitions between bubble states is needed to gain a physical understanding of bubble selection and propagation. Moreover, the observations reported in this letter set new challenges for the modeling of airway reopening.

The authors wish to thank Dr. A. L. Hazel and Professor T. Mullin for helpful discussions. This work was funded by an EPSRC studentship (A.H.) and an EPSRC "Advanced Research Fellowship" (A.J.).

${ }^{1}$ M. Joanicot and A. Adjari, "Droplet control for microfluidics," Science 309, 887 (2005).

${ }^{2}$ W. L. Olbricht, "Pore-scale prototypes of multiphase flow in porous media," Annu. Rev. Fluid Mech. 28, 187 (1996).

${ }^{3}$ W. A. Hodson, "The first breath," in The Lung: Scientific Foundations, edited by R. G. Crystal, J. B. West, P. J. Barnes, and E. R. Weibel (Lippincott, Williams, and Wilkins, New York, 1991).

${ }^{4}$ J. M. B. Hughes, D. Y. Rosenzweig, and P. B. Kivitz, "Site of airway closure in excised dog lungs: Histologic demonstration," J. Appl. Physiol. 29, 340 (1970).

${ }^{5}$ P. T. Macklem, D. F. Proctor, and J. C. Hogg, "The stability of peripheral airways," Respir. Physiol. 8, 191 (1970).

${ }^{6}$ C. C. Dos Santos and A. S. Slutsky, "Invited review: Mechanisms of ventilator-induced lung injury: A perspective," J. Appl. Physiol. 89, 1645 (2000).

${ }^{7}$ E. T. Naureckas, C. A. Dawson, B. S. Gerber, D. P. Gaver III, H. L. Gerber, J. M. Lineran, J. Solway, and R. W. Samsel, "Airway reopening pressure in isolated rat lungs," J. Appl. Physiol. 76, 1372 (1994).

${ }^{8}$ J. B. Grotberg and O. E. Jensen, "Biofluid mechanics in flexible tubes," Annu. Rev. Fluid Mech. 36, 121 (2004).

${ }^{9}$ A. L. Hazel and M. Heil, "Three-dimensional airway reopening: The steady propagation of a semi-infinite bubble into a buckled elastic tube," J. Fluid Mech. 478, 47 (2003).

${ }^{10}$ E. Lorenceau, F. Restagno, and D. Quéré, "Fracture of a viscous liquid," Phys. Rev. Lett. 90, 184501 (2003).

${ }^{11}$ S. Courrech du Pont and J. Eggers, "Sink flow deforms the interface between a viscous liquid and air into a tip singularity," Phys. Rev. Lett. 96, 034501 (2006)

${ }^{12}$ I. Cohen and S. R. Nagel, "Scaling at the selective withdrawal transition through a tube suspended above the fluid surface," Phys. Rev. Lett. 88, 074501 (2002).

${ }^{13}$ A. Juel and A. Heap, "The reopening of a collapsed fluid-filled elastic tube," J. Fluid Mech. 572, 287 (2007).

${ }^{14}$ M. Heil, A. L. Hazel, and J. A. Smith, "The mechanics of airway closure," Respir. Physiol. Neurbiol. (in press).

${ }^{15}$ D. P. Gaver III, R. W. Samsel, and J. Solway. "Effects of surface tension and viscosity on airway reopening," J. Appl. Physiol. 69, 74 (1990).

${ }^{16}$ M. L. Perun and D. P. Gaver III, "An experimental model investigation of the opening of a collapsed untethered pulmonary airway," ASME J. Biomech. Eng. 117, 245 (1995).

${ }^{17}$ D. P. Gaver III, D. Halpern, O. E. Jensen, and J. B. Grotberg, "The steady motion of a semi-infinite bubble through a flexible-walled channel," J. Fluid Mech. 319, 25 (1996).

${ }^{18}$ J. E. Flaherty, J. B. Keller, and S. I. Rubinow, "Post-buckling behaviour of elastic tubes and rings with opposite sides in contact," SIAM J. Appl. Math. 23, 446 (1972).

${ }^{19}$ A. L. Hazel and M. Heil, "Finite-Reynolds-number effects in steady, threedimensional airway reopening," ASME J. Biomech. Eng. 128, 573 (2006).

${ }^{20}$ A. Heap, "The reopening of a collapsed, fluid-filled elastic tube," Ph.D. thesis, University of Manchester, 2008.

${ }^{21}$ M. Heil, "Minimal liquid bridges in non-axisymmetrically buckled elastic tubes," J. Fluid Mech. 380, 309 (1999).

${ }^{22}$ A. L. Hazel and M. Heil, "The steady propagation of a semi-infinite bubble into a tube of elliptical or rectangular cross-section," J. Fluid Mech. 470, 91 (2002).

${ }^{23} \mathrm{P}$. Petitjeans and T. Maxworthy, "Miscible displacements in capillary tubes. Part I. Experiments,” J. Fluid Mech. 326, 37 (1996). 